\title{
Safety Profile and Clinical Recommendations for the Use of Lapatinib
}

\author{
Dimitrios Chatsiproios \\ Gynäkologisch-onkologische Praxis Kreuzlingen, Switzerland
}

\author{
Key Words \\ Lapatinib - Tolerability · Rash · Diarrhea · \\ Clinical management
}

\section{Summary}

The toxicity profile of agents blocking the epidermal growth factor receptor pathway excludes many of the severe side effects commonly observed with cytotoxic chemotherapy. Like other tyrosine kinase inhibitors, lapatinib is generally well tolerated. Lapatinib exhibits specific toxicities, the most common being diarrhea and rash, which are mostly mild to moderate in severity. Cardiac toxicity is rarely seen with lapatinib. Generally, lapatinib-associated toxicities are manageable.

\section{Introduction}

Contrary to standard chemotherapy agents which affect most replicating cells, drug-induced blockade of ErbB family members targets specific signaling pathways that are essential for tumor cell proliferation and survival. Therefore, treatment with these targeted therapies excludes many of the severe systemic and particularly hematopoietic side effects often seen with cytotoxic chemotherapy. However, as anti-ErbB1 treatment is increasingly part of the therapeutic armamentarium for the treatment of different malignancies, commonly occurring adverse events blocking the ErbB1 pathway have been identified. These reactions are caused by coincident inhibition of receptor activity in tissues that depend on ErbB1 signaling

\author{
Schlüsselwörter \\ Lapatinib · Verträglichkeit · Hautveränderungen · \\ Diarrhö · Klinisches Management
}

\section{Zusammenfassung}

Unter Anti-EGFR(epidermal growth factor receptor)-Therapeutika treten viele der schweren Nebenwirkungen nicht auf, die häufig unter Chemotherapie gesehen werden. Wie andere Tyrosinkinasehemmer wird auch Lapatinib allgemein gut vertragen. Zu den häufigsten Nebenwirkungen gehören Hautveränderungen («Rash») und Diarrhö, die beide meist von leichtem bis moderatem Schweregrad sind. Kardiale Toxizitäten sind unter Lapatinib nur selten. Generell lassen sich die Lapatinib-assoziierten Nebenwirkungen gut beherrschen.

for physiological function. ErbB1 is expressed on nearly all body cells, particularly those of epithelial origin such as skin, liver, and the gastrointestinal tract, but not on hematopoietic cells $[1,2]$. As a consequence, the most commonly encountered toxic effects from agents blocking the epidermal growth factor receptor (EGFR) are rash and diarrhea, as well as hepatic disorders. ErbB2 (HER2)- and ErbB1-inhibiting therapies have also resulted in cardiotoxicity. Yet, these adverse events appear to differ in frequency and severity within the various ErbB1-targeting agents. In conclusion, awareness as well as improved understanding and proper management of these side effects are crucial in order to increase patients' compliance, adherence and persistence, and to ensure quality of life and overall treatment success.

\section{KARGER}

Fax +497614520714

Information@Karger.de

www.karger.com (c) 2010 S. Karger GmbH, Freiburg 


\section{Tolerability in Clinical Phase III Trials}

The safety of lapatinib has been evaluated in clinical trials involving more than 3,500 patients [3]. The most frequently reported drug-related adverse events $(>20 \%)$ in the pivotal phase III trial EGF 100151 with lapatinib plus capecitabine were gastrointestinal (diarrhea, nausea, and vomiting), dermatologic (palmar-plantar erythrodysesthesia and rash), and fatigue. In the combination arm, $60 \%$ of women experienced diarrhea (27\% grade $1,20 \%$ grade $2,12 \%$ grade 3 , and $1 \%$ grade 4 ), versus $39 \%$ in the capecitabine monotherapy arm ( $14 \%$ grade $1,14 \%$ grade $2,11 \%$ grade 3$)(p<0.001)$ [4]. In the combination group, $27 \%$ of patients developed rash compared to $15 \%$ in the capecitabine monotherapy arm ( $\mathrm{p}=$ 0.011 ) [4]. The vast majority of rash cases were mild to moderate (grade 1 or 2 ), with grade 3 rash seen in $1 \%$ of patients in both the combination arm and the capecitabine monotherapy arm. As compared with capecitabine alone, lapatinib plus capecitabine was not associated with an increase in either serious toxic effects or rates of discontinuation related to adverse events. Adverse events led to discontinuation of treatment in 22 women in the combination therapy group $(13 \%)$ and in 18 women in the monotherapy group (12\%). There were no differences in the mean left ventricular ejection fraction (LVEF) by echocardiography or multiple gated acquisition (MUGA) scanning between the two groups at scheduled assessments in the EGF 100151 trial [4]. In the combination arm, 4 women experienced asymptomatic and reversible cardiac dysfunction considered to be treatmentrelated, while 1 patient in the capecitabine monotherapy group suffered from a cardiac event unrelated to treatment, that remained unresolved. No symptomatic cardiac events were observed, and there were no withdrawals from treatment due to declines in LVEF, no cases of congestive heart failure (CHF), and no decreases in the mean LVEF values in the group receiving lapatinib.

This safety profile of the small-molecule dual tyrosine kinase inhibitor (TKI) was confirmed by the data of the second phase III trial evaluating the efficacy of lapatinib in combination with paclitaxel as first-line therapy in 579 women with metastatic breast cancer (EGF 30001) [5]. Diarrhea was the most common adverse event, experienced by $58 \%$ of women in the combination arm and $26 \%$ in the paclitaxel monotherapy group $(\mathrm{p}<0.0001)$. Rash was seen in $43 \%$ of women in the lapatinib/paclitaxel arm versus $21 \%$ receiving paclitaxel monotherapy $(\mathrm{p}<0.0001)$. Lapatinib combined with paclitaxel was associated with a $2.7 \%$ incidence of fatal adverse events (mainly sepsis associated with diarrhea), compared with a $0.6 \%$ incidence in the paclitaxel/placebo arm. According to the authors, most of these fatal events occurred early in the accrual period and decreased sharply with time. Increased experience treating these adverse events and the introduction of proactive guidelines for managing lapatinib-related diarrhea during the study likely contributed to the reduced in- cidence and severity of diarrhea episodes. Six patients $(2 \%)$ in each treatment group of the EGF 30001 trial had a decrease in LVEF [5]. Five of 6 patients in each group experienced an asymptomatic LVEF decrease that met protocoldefined serious adverse event (SAE) criteria. None of these events required a dose/schedule adjustment or resulted in treatment withdrawal or death. Decreases in LVEF were considered treatment-related in 3 of 6 patients in the paclitaxel/lapatinib arm and in 4 of 6 patients in the paclitaxel/placebo group. All events occurred more than 28 days after treatment initiation.

\section{Diarrhea and Clinical Management}

To characterize diarrhea events in cancer patients treated with lapatinib as monotherapy or in combination with capecitabine or taxanes, Crown et al. [8] conducted a pooled analysis of 9 clinical trials (phase I, II, or III) in patients with metastatic cancer. Lapatinib was administered at doses ranging from 1,000 to $1,500 \mathrm{mg} /$ day as monotherapy $(\mathrm{n}=926)$ or in combination with capecitabine $(\mathrm{n}=198)$ or taxanes $(\mathrm{n}=$ 687). Diarrhea events were characterized based on severity, time to onset, duration, required interventions, and clinical outcomes. Diarrhea occurred in 55\% of lapatinib-treated patients and $24 \%$ of patients not receiving lapatinib. All-grade diarrhea experienced $51 \%$ of patients treated with lapatinib monotherapy and $65 \%$ under lapatinib plus capecitabine. In a separate analysis, $48 \%$ of women receiving lapatinib plus a taxane experienced diarrhea. Overall, most diarrhea events were low-grade $1 / 2$, requiring infrequent lapatinib dose modification or interruption. Grade 3 events occurred in $<10 \%$ of patients, and grade 4 events were rare $(<$ or $=1 \%)$. Most diarrhea events resolved with conventional approaches and without dose modification. Approximately $40 \%$ of patients treated with lapatinib monotherapy or combination therapy experienced a first diarrhea event within 6 days of treatment initiation, with a median duration of 7-9 days. Lapatinibcontaining chemotherapy regimens did not cause severe diarrhea when proactive monitoring and intervention was introduced.

Management of diarrhea is crucial to prevent more serious complications in lapatinib-treated patients. Therefore, Chatsiproios and Jackisch [from 9-14] developed a useful algorithm which can be applied by nurses/office assistants (fig. 1) and physicians (fig. 2). If a patient experiences mild to moderate (grade 1 or 2 ) diarrhea, dietary measures like avoiding all lactose-containing products, staying well hydrated, eating frequent small meals, as well as administering standard doses of loperamide are indicated. Diarrhea has to be controlled after $24 \mathrm{~h}$. If there is no improvement seen after $24 \mathrm{~h}$, if the patient experiences severe diarrhea or has complicating features such as severe cramping, severe nausea or vomiting, fever or dehydration, she has to be seen by a physician, and inpatient hospi- 
Fig. 1. Management algorithm for lapatinibassociated diarrhea for nurses/office assistants developed by the author and C. Jackisch from [9-14].

Fig. 2. Management algorithm for lapatinibassociated diarrhea for physicians developed by the author and C. Jackisch from [9-14].
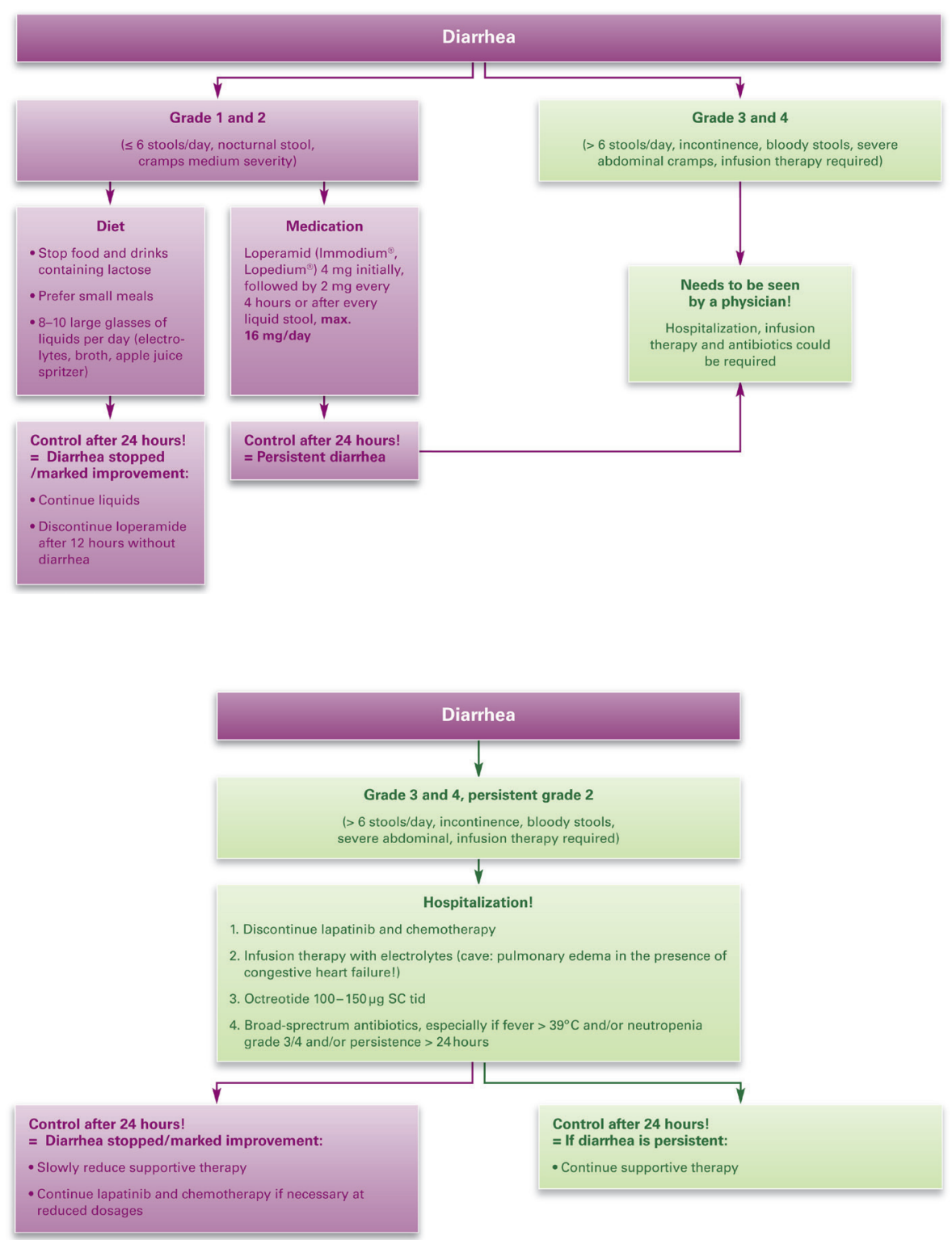

talization should be considered (fig. 2). Further doses of lapatinib and chemotherapy should be held; intravenous (IV) fluids, octreotid 100-150 $\mu \mathrm{g}$ subcutaneously 3 times daily (not licensed in Germany for this indication), and antibiotics might be required. Repeated administration of lapatinib should be delayed until the patient recovers.

\section{Rash and Clinical Management}

Skin reactions are common in patients receiving ErbB1-targeted treatments, regardless of therapy type (i.e. ErbB1 TKIs or monoclonal antibodies). ErbB1 activation mediates essential functions in the skin such as the promotion of keratinocyte proliferation, survival and motility, and the regulation of differentiation and keratinization [15]. In wounded skin, the ErbB1 receptor is transiently up-regulated, and is an important contributor to the proliferative and migratory aspects of wound re-epithelialization. Therefore, blocking the ErbB1 pathway at the skin level can result in abnormal proliferation, migration, and differentiation of target cells, and consequent disruption of the integrity of the skin with recruitment of inflammatory cells. The most commonly seen skin reaction with ErbB1 inhibitors is a follicular acneiform eruption, also termed acne-like rash or folliculitis, characterized by inflammatory papules and pustules most often seen on the face, chest, and back [3]. The distribution is called 'acneiform' because the lesions are present at sites with large numbers of pilosebaceous units such as the scalp, face, neck, and upper trunk. However, the characteristic rash is different from classic acne vulgaris because of its lack of comedones. 
Fig. 3. Management algorithm for lapatinibassociated rash for nurses/office assistants adapted by the author and C. Jackisch from [18].
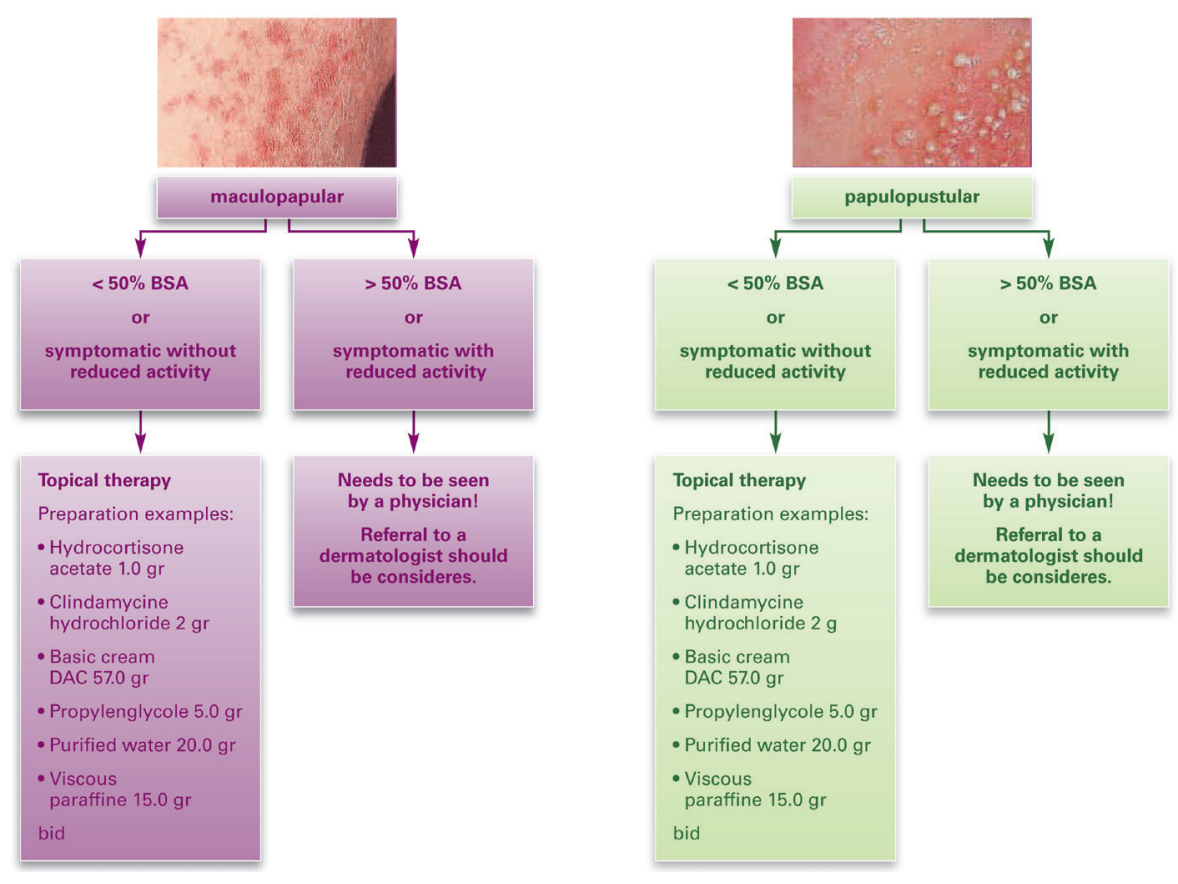

Fig. 4. Management algorithm for lapatinibassociated rash for physicians adapted by the author and C. Jackisch from [18].
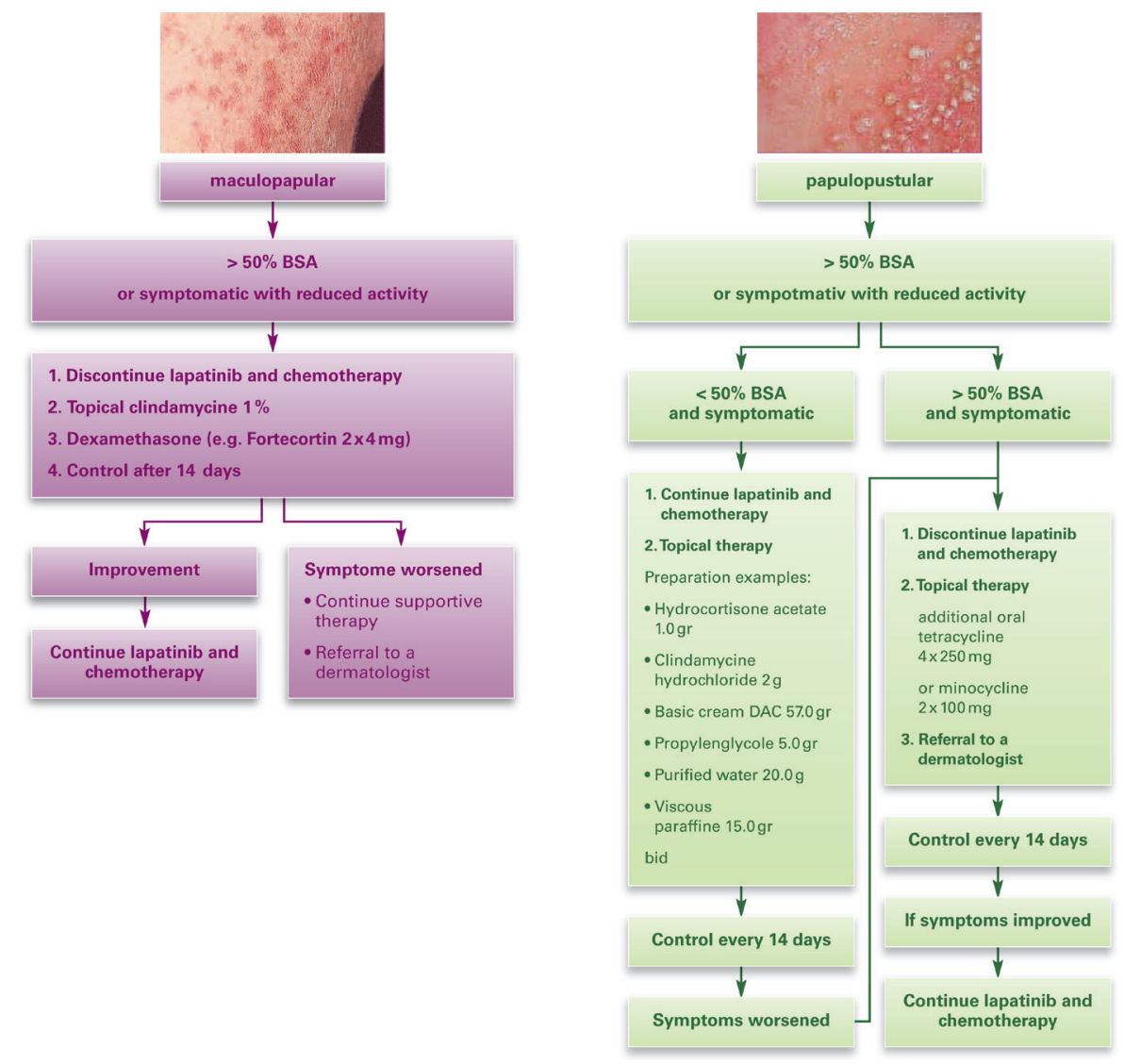

Rash associated with ErbB1 inhibitors generally follows a well-characterized clinical course: Within the first week of treatment, patients experience sensory disturbance with erythema and edema [16]. From weeks 1 to 3, the papulopustular eruption becomes manifest, followed by crusting at week 4 . Despite successful treatment, erythema and dry skin may persist in the areas previously affected by the skin rash through weeks 4 to 6 . Other less frequent dermatological side effects 
Table 1. Potential drug-drug interactions with lapatinib, adapted from [3]

\begin{tabular}{|c|c|}
\hline Drug class & Agent \\
\hline \multicolumn{2}{|l|}{ CYP3A4 inducers } \\
\hline Antibiotics & all rifamycin class agents (e.g. rifampicin, rifabutin, rifapentine) \\
\hline Anticonvulsants & phenytoin, carbamazepine, barbiturates (e.g. phenobarbital) \\
\hline Antiretrovirals & efavirenz, nevirapine \\
\hline Glucocorticoids (oral) & $\begin{array}{l}\text { cortisone }(>50 \mathrm{mg}) \text {, hydrocortisone }(>40 \mathrm{mg}) \text {, prednisone }(>10 \mathrm{mg}) \text {, methyl- } \\
\text { prednisone }(>8 \mathrm{mg}) \text {, dexamethasone }(>1.5 \mathrm{mg})\end{array}$ \\
\hline Other & St. John's Wort, modafinil \\
\hline \multicolumn{2}{|l|}{ CYP3A4 inhibitors } \\
\hline Antibiotics & clarithromycin, erythromycin, troleandomycin \\
\hline Antifungals & itraconazole, ketoconazole, fluconazole (>150 mg daily), voriconazole \\
\hline Antiretrovirals, protease inhibitors & delaviridine, nelfinavir, amprenavir, ritonavir, indinavir, saquinavir, lopinivir \\
\hline Calcium channel blockers & verapamil, diltiazem \\
\hline Antidepressants & nefazodone, fluvoxamine \\
\hline Gastrointestinal agents & cimetidine, aprepitant \\
\hline Other & grapefruit, grapefruit juice, amiodarone \\
\hline \multicolumn{2}{|l|}{ Miscellaneous } \\
\hline Antacids & Mylanta $^{\circledR}$, Maalox $^{\circledast}$, Tums $^{\circledast}$, Rennies $^{\circledast}(1 \mathrm{~h}$ before and after lapatinib dosing \\
\hline Herbal supplements & gingko biloba, kava, grape seed, valerian, ginseng, echinacea, evening primrose oil \\
\hline
\end{tabular}

include dry skin, pruritus, fissures, palmar-plantar rash, hyperkeratosis, and nail disorders.

In an overview of dermatologic events (DEs) in patients with metastatic cancer treated with lapatinib, Lacouture et al. [17] analyzed 9 clinical trials. Lapatinib was administered at doses ranging from 1,000 to $1,500 \mathrm{mg}$ /day as monotherapy $(\mathrm{n}=928)$ or in combination with paclitaxel or capecitabine $(n=491)$. Patients not treated with lapatinib comprised the control group. Dermatologic events included hand-foot syndrome, rash, hair disorder, dry skin, pruritus/urticaria, skin disorder, skin infection, and nail disorder. Overall, 58\% of patients receiving lapatinib monotherapy, $74 \%$ of patients treated with lapatinib plus paclitaxel or capecitabine, and $53 \%$ of patients in the control group developed dermatologic events [17]. Among patients receiving lapatinib monotherapy, $55 \%$ experienced grade $1 / 2$ DEs, $3 \%$ had grade 3 DEs. No grade 4 DEs were observed. The most common event was rash $(43 \%)$; all other events occurred in $\leq 8 \%$ of patients. Most dermatologic side effects developed between days 1 and 14 after starting treatment, with a median duration of 29 days. In most patients, rash attributable to lapatinib resolved during treatment, following a temporary interruption in treatment, or after treatment cessation. A total of $3 \%$ of DEs led to a dose reduction of lapatinib, $7 \%$ resulted in dose interruption, and only $1 \%$ led to drug discontinuation. Compared to other ErbB1 inhibitors, lapatinib is associated with fewer and less severe skin events [17].

There are no clear evidence-based recommendations for management of lapatinib-associated rash. However, there is considerable experience in managing dermatologic events from other ErbB1-targeted agents [16]. An effective multidis- ciplinary management as well as patient education are paramount to improve the symptoms and optimize clinical outcomes by minimizing the need for significant dose reductions or discontinuation. In general, patients should be advised to take appropriate sun protective measures, because sunexposure can exacerbate rash severity on unprotected areas of the body. In addition, greasy ointments and skin care products that exsiccate the skin should be avoided. Instead, frequent moisturizing with alcohol-free emollient creams is recommended.

For the management of skin rash, it is crucial to distinguish between papulopustular and maculopapular eruptions, to determine the extent of body surface area (BSA) affected, and to consider the severity of symptoms (fig. 3 and 4) [18]. Patients with less than $50 \%$ BSA and symptoms that do not affect activities of daily living can be treated with topical medications. If more than $50 \%$ BSA is affected, lapatinib and chemotherapy should be discontinued, and topical therapy should be given. Topical retinoids, like the antiacne medication tretinoin, are generally not recommended, because rash seen with lapatinib has a different pathophysiology than acne vulgaris [3]. Referral to a dermatologist is recommended if the skin toxicity does not improve within 1-2 weeks of treatment, or if the patient is severely symptomatic [16].

\section{Cardiac Function and Clinical Management}

Because of the cardiac toxicity associated with trastuzumab $[19,20]$, Perez et al. [21] analyzed the cardiac safety of lapatinib using prospective data collected in 44 clinical phase I, II, 
and III studies. These data showed a very low rate of symptomatic CHF $(0.2 \%)$ or asymptomatic cardiac events $(1.4 \%)$ [21]. None of the 53 asymptomatic patients received cardiacrelated treatment; no cardiac deaths were attributed to lapatinib. As concluded by the authors, this review of data from 44 clinical studies revealed low levels of cardiotoxicity for lapatinib [21]. However, given the similarities between trastuzumab- and lapatinib-induced cardiac toxicity, the cardiac safety consensus guidelines for the adjuvant use of trastuzumab of the Cardiac Guidelines Consensus Committee can serve as a basis also for patients on lapatinib therapy $[3,22]$. Before initiating lapatinib, the baseline LVEF evaluated by MUGA scan or echocardiography should be $\geq 50 \%$. Subsequent cardiac evaluations are recommended, using the same method as in the baseline evaluation [22]. Lapatinib administration should generally be avoided in patients with a subnormal LVEF.

\section{Drug Interactions}

The elimination of lapatinib occurs through hepatic metabolism primarily through cytochrome P450 (CYP)3A4 and excretion in feces [3]. Lapatinib inhibits CYP3A4 and CYP2C8 in vitro at clinically relevant concentrations [23]. Therefore, caution should be exercised when dosing lapatinib concurrently with medications with narrow therapeutic windows that are substrates of CYP3A4 or CYP2CA8 (table 1) [3, 23]. Lapatinib undergoes extensive metabolism by CYP3A4. Concomitant administration of inducers or strong inhibitors of CYP3A4 significantly alter lapatinib concentrations (table 1) and should be avoided [23]. Antacids that modify gastric $\mathrm{pH}$ may affect the absorption of lapatinib and should generally be avoided for $1 \mathrm{~h}$ before and after lapatinib dosing.

\section{Disclosure Statement}

The author was a speaker and contributor to the meeting 'ErbB2 (HER2)-positives Mammakarzinom; 2. Münchner Brustkrebs-Symposium Update 2009', 23/24 October 2009 in Munich, sponsored by GlaxoSmithKline

\section{References}

1 O'Keefe E, Battin T, Payne T Jr: Epidermal growth factor receptor in human epidermal cells direct demonstration in cultured cells. J Invest Dermatol 1982;78:482-487.

2 Harandi A, Zaida A, Stocker AM, Laber D: Clinical efficacy and toxicity of anti-EGFR therapy in common cancers. J Oncol 2009; Epub ahead of print.

3 Moy B, Goss PE: Lapatinib-associated toxicity and practical management recommendations. Oncologist 2007;12:756-765.

$\checkmark 4$ Geyer CE, Forster J, Lindquist D, et al.: Lapatinib plus capecitabine for HER-2-positive advanced breast Cancer. N Engl J Med 2006;355:2733-2743.

5 Di Leo A, Gomez HL, Aziz Z, et al.: Phase III, double-blind, randomized study comparing lapatinib plus paclitaxel with placebo plus paclitaxel as first-line treatment for metastatic breast cancer. J Clin Oncol 2008:26:5544-5552.

6 Crown JP, Burris HA, Jones S, et al.: Safety and tolerability of lapatinib in combination with taxanes $(\mathrm{T})$ in patients with breast cancer (BC). J Clin Oncol 2007;25(suppl):38s, abstr 1027.

7 Jones SF, Burris HA 3rd, Yardley DA, et al.: Lapatinib (an oral dual kinase inhibitor) plus weekly or every 3 week paclitaxel. 27th Annual San Antonio Breast Cancer Symposium 2004;abstr 1069.

-8 Crown JP, Burris HA 3rd, Boyle F, et al.: Pooled analysis of diarrhea events in patients with cancer treated with lapatinib. Breast Cancer Res Treat 2008;112:317-325. $\checkmark$ Arbuckle RB, Huber SL, Zacker C: The consequences of diarrhea occurring during chemotherapy for colorectal cancer: a retrospective study. Oncologist 2000;5:250-259.

10 Benson AB, Ajani JA, Catalano RB: Recommended guidelines for treatment of cancer treatment-induced diarrhea. J Clin Oncol 2004;22:291826.

11 Cascinu S, Barni S, Labianca R: Evaluation of factors influencing 5-fluorouracil-induced diarrhea in colorectal cancer patients. An Italian Group for the Study of Digestive Tract Cancer study. Support Care Cancer 1997;5:314-317.

12 Rothenberg ML, Meropol NJ, Poplin EA: Mortality associated with irinotecan plus bolus fluorouracil/leucovorin: summary findings of an independent panel. J Clin Oncol 2110;19:3801-3807.

13 Rosenoff S: Octreotide LAR resolves severe chemotherapy-induced diarrhea and allows continuation of full-dose therapy. Proc Am Soc Oncol 2003:22:778(abstr 3126)

14 Wadler S, Benson AB, Engelking C: Recommended guidelines for the treatment of cheomtherapyinduced diarrhea. J Clin Oncol 1998;16:3169-3178.

15 Segaert S, Van Cutsem E: Clinical signs, pathophysiology and management of skin toxicity during therapy with epidermal growth factor receptor inhibitors. Ann Oncol 2005;16:1425-1433.
16 Melosky B, Burkes R, Rayson D, Alcindor T, Shear N, Lacouture M: Management of skin rash during EGFR-targeted monoclonal antibody treatment for gastrointestinal malignancies: Canadian recommendations. Curr Oncol 2009;16:16-26.

17 Lacouture ME, Laabs SM, Koehler M, et al. Analysis of dermatologic events in patients with cancer treated with lapatinib. Breast Cancer Res Treat 2009;114:485-493.

18 Moy B and Goss PE: Lapatinib associated toxicity and practical management recommendations. Oncologist 2007;12:756-765.

19 Perez EA: Cardiac toxicity of ErbB2-targeted therapies: what do we know? Clin Breast Cancer 2008;8(suppl 3):S114.

20 Slamon DJ, Leyland-Jones B, Shak S, et al.: Use of chemotherapy plus a monoclonal antibody against HER2 for metastatic breast cancer that overexpresses HER2. N Engl J Med 2001;344:783-792.

21 Perez EA, Koehler M, Byrne J, Preston AJ, Rappold E, Ewer MS: Cardiac safety of lapatinib: pooled analysis of 3689 patients enrolled in clinical trials. Mayo Clin Proc 2008;83:679-686.

22 Ewer MS, Perez EA, Baselga J, et al.: Cardiac safety guidelines for the adjuvant use of trastuzumab (Herceptin) in HER2-positive early breast cancer. Breast 2007;16(suppl 1):S63.

23 Tyverb® full prescribing information, August 2009; us.gsk.com/products/assets/us_tykerb.pdf. 